\title{
Primary pleural epithelioid haemangioendothelioma with metastases to the skin. A case report and literature review
}

\author{
M Al-Shraim, B Mahboub, P C Neligan, D Chamberlain, D Ghazarian
}

J Clin Pathol 2005;58:107-109. doi: 10.1136/icp.2004.018937

Epithelioid haemangioendothelioma (EHE) is a rare vascular tumour of intermediate behaviour. It can arise from various sites including the liver, spleen, pleura, or lung. Cutaneous EHE can be primary or secondary. This report describes the case of a 51 year old man who presented with a history of dry cough, shortness of breath, and pleural effusion, and who developed two cutaneous nodules in the anterior abdominal wall a few weeks later. He had a previous history of asbestos exposure. Computed tomography scan showed a left sided pleural effusion and nodular pleural mass. Histology of both the pleural and cutaneous lesions was compatible with EHE. Electron microscopic examination demonstrated the presence of Weibel-Palade bodies. The patient underwent elliptical excision of the metastatic cutaneous nodules after decortication of the primary pleural tumour and adjuvant treatment. A few reports have described metastasis of intrathoracic EHE to the skin. Despite treatment with interferon, the patient developed more cutaneous lesions two years after the initial diagnosis. Even though the tumour has the classic light histological and ultrastructural features of $\mathrm{EHE}$, it behaved in an aggressive manner.

\footnotetext{
A 51 year old man presented with a history of dry cough, which was treated initially with bronchodilator, but with no improvement; one week later he developed shortness of breath on exertion. Chest $x$ ray showed a pronounced left sided pleural effusion. The bronchoscopic examination was unremarkable. He underwent pleuroscopy with multiple pleural biopsies, and the results were interpreted at his regional hospital as malignant mesothelioma, undifferentiated, small cell type. His past medical history revealed exposure to asbestos for two to three months about 25 years previously. The patient was referred to Princess Margaret Hospital, Toronto, Canada, for further management. The patient received one cycle of vinorelbine and cisplatin based on the presumed diagnosis of mesothelioma. However, the pathology was reviewed at that point by the pulmonary pathologist, and a revised diagnosis of epithelioid haemangioendothelioma (EHE) was made. On examination, the patient had no cervical lymphadenopathy. Respiratory assessment revealed greatly decreased chest expansion, in addition to decreased air entry on the left slide. He had dullness on percussion of his left lung posteriorly compared with the right lung. A thoracic computed tomography (CT) scan showed a moderately sized pleural effusion on the left side. There was pleural thickening, which became a nodular mass up to $6.2 \mathrm{~cm}$ in greatest dimension (fig 1). Multiple gas loculations were noted within the pleural fluid. He had a left lung decortication and resection of the pleural tumour.
}

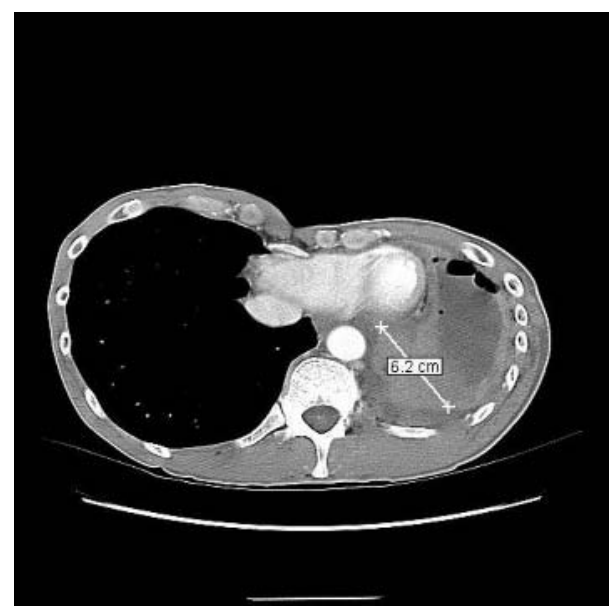

Figure 1 Computed tomography scan showing pleural thickening, a nodular mass in the inferior left pleural surface, and a pleural effusion with multiple gas loculations.

Light microscopic examination of the pleural tumour showed strips of dense fibrous tissue, with focal infiltration by nests and trabeculae of epithelioid cells. These cells showed irregularly round and rather uniform appearing nuclei, with open chromatin and a single prominent nucleolus. Many tumour cells showed cytoplasmic vacuoles or lumens, some of which had a bubble-like appearance (fig 2A). The tumour showed abundant eosinophilic stroma with focal areas of necrosis. These tumour cells showed moderate and diffuse (approximately 95\%) immunoreactivity for CD31 (fig 3A) and factor VIII, strong and diffuse (approximately 95\%) immunoreactivity for CD34 (fig 3B), strong and diffuse (approximately $80 \%$ ) immunoreactivity for monoclonal carcinoembryonic antigen (CEA; fig 3C) and vimentin (fig 3D), and weak and focal immunoreactivity (approximate 5\%) for cytokeratin 7. No immunoreactivity for thyroid transcription factor 1 or BerEP4 was seen. Electron microscopic examination revealed Weibel-Palade bodies (fig 4). The patient was started on three subcutaneous injections of 7000000 units of interferon $\alpha$ each week. A few weeks later he developed small, multiple, subcutaneous nodules on his anterior abdominal wall. They were firm, mobile, and mildly tender to pressure. The patient underwent elliptical excision of these subcutaneous nodules. These skin lesions had an identical histology to the primary pleural tumour (fig 2B) and showed a similar immunoreactive pattern for CD31, CD34, vimentin, and monoclonal CEA. Serial thoracic CT scans showed no interval change in the size of the residual tumour over a follow up period of two years. Abdominal CT scans showed moderate pelvic ascites, which

Abbreviations: CEA, carcinoembryonic antigen; $\mathrm{CT}$, computed tomography; EHE, epithelioid haemangioendothelioma 

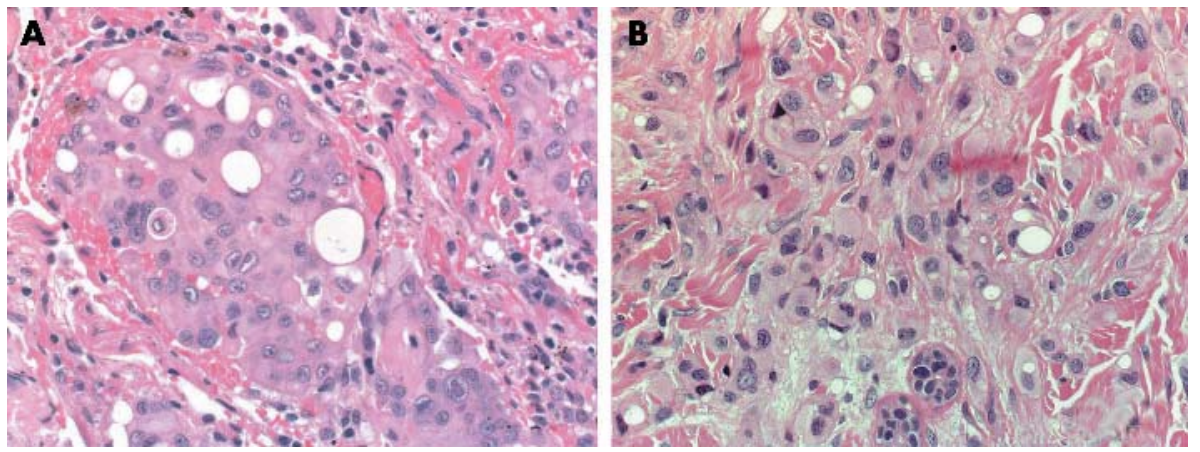

Figure 2 (A) Haematoxylin and eosin (H\&E) section of pleura disclosing trabeculae of epithelioid tumour cells with cytoplasmic vacuoles and lumens (original magnification, $\times 200$ ); (B) H\&E section of skin showing epithelioid haemangioendothelioma cells separated by collagen fibres (original magnification, $\times 200$ ).

were unchanged from the previous scans. The patient continued on interferon treatment. More recently he has developed more cutaneous nodules on his trunk.

\section{DISCUSSION}

EHE is a rare vascular tumour of intermediate behaviour, which was first described by Weiss and Enzinger in 1982. ${ }^{1}$ It can originate from the soft tissue, lung, pleura, liver, bone, breast, brain, or meninges. ${ }^{1-3}$ It is extremely rare for EHE to arise from the skin as a primary tumour. ${ }^{4}$ We describe the case of a 51 year old man who developed multiple metastatic cutaneous lesions of EHE from a pleural primary location. He had a history of exposure to asbestos. A histological and immunopathological examination of both the lesions was compatible with EHE. The tumour cells stained negatively for mesothelium associated markers. The diagnosis of EHE was confirmed by the demonstration of Weibel-Palade bodies on electron microscopy. The association between exposure to asbestos and the development of several pleural lesions including malignant mesothelioma, hyaline plaques, and diffuse pleural fibrosis has been well established..$^{5-7}$ However, the patient's exposure to asbestos was short and he had no radiological features, such as pleural plaques; in addition, the association between asbestos and pleural epithelioid haemangioendothelioma has been postulated only in one series of three patients. ${ }^{8}$ EHE can mimic malignant mesothelioma clinically, radiologically, ${ }^{9}$ and histomorphologically, ${ }^{8}$ so that EHE should be suspected in any lesion of pleural malignant mesothelioma, because the management and the prognosis of these two diseases are different. On light microscopic examination, EHE can mimic mesothelioma; however, immunohistochemical staining for vascular markers such as CD31, CD34, and factor VIII can help to distinguish it from mesothelioma. ${ }^{10-12} \mathrm{~A}$ few reports described the metastasis of primary intrathoracic EHE to the skin. ${ }^{13-15}$ The cutaneous lesions seen in our patient showed a similar histology; this was interpreted as a metastatic lesion from the primary pleural lesion.

The expression of monoclonal CEA has been investigated by immunohistochemical studies as a prognostic marker of the biological aggressiveness of many human tumours, including thymic carcinomas, ${ }^{16}$ colorectal carcinomas, ${ }^{17}$ synovial sarcomas, ${ }^{18}$ and others. ${ }^{19}{ }^{20}$ The expression of monoclonal CEA in our case is an unusual and interesting finding, which might be related to the aggressive course of this vascular tumour. However, its prognostic value for EHE remains a subject for further investigations. According to the literature, EHE is negative for monoclonal CEA. This is based on only a few rare reported cases. ${ }^{21}$ We recently noticed that some

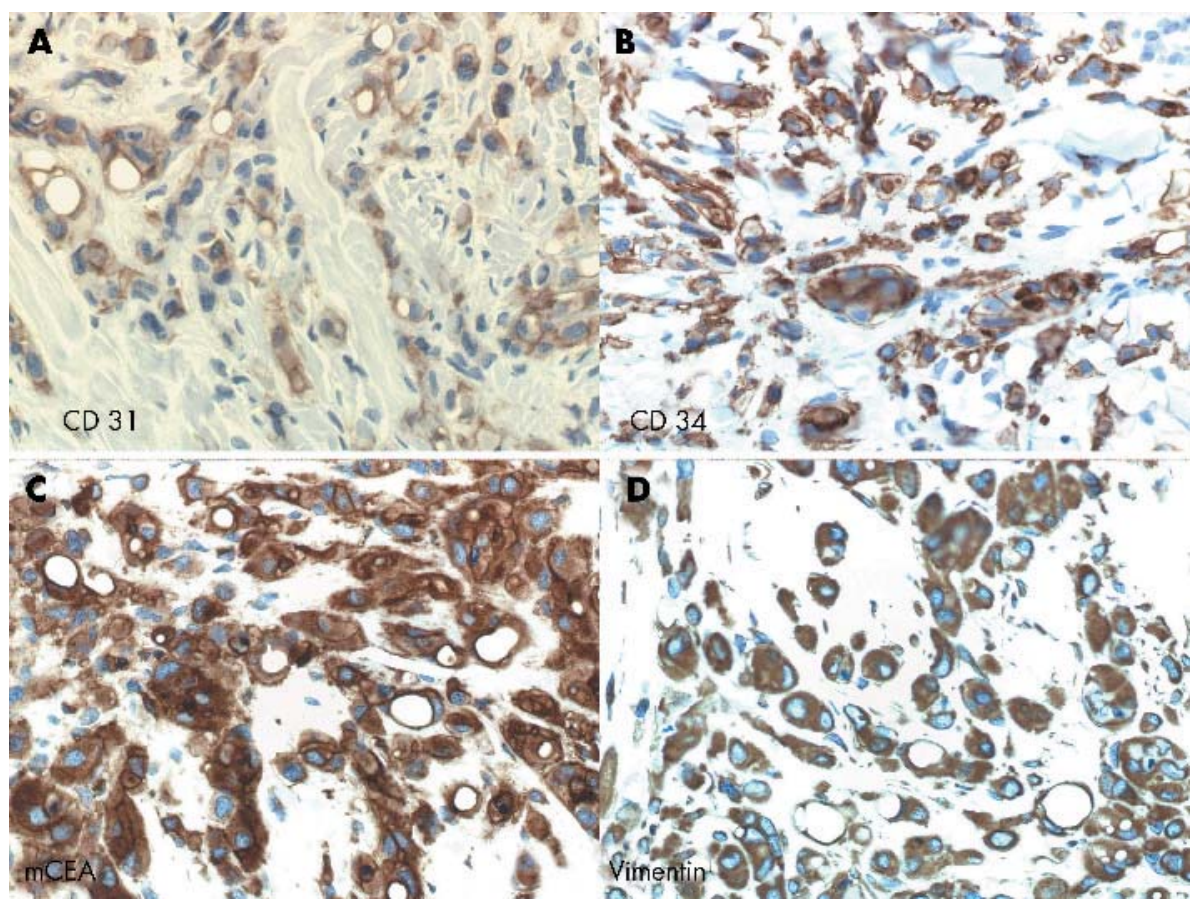

Figure 3 Immunoperoxidase staining of the tumour showing positive immunoreactivity for (A) CD31, (B) CD34, (C) monoclonal carcinoembryonic antigen, and (D) vimentin. 


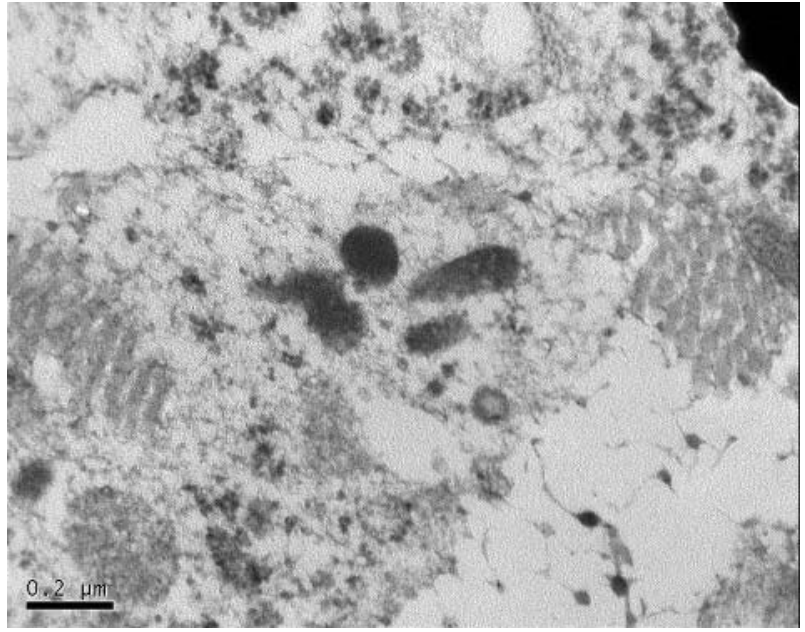

Figure 4 Electron micrograph showing Weibel-Palade bodies.

haemangioendotheliomas stain positively for monoclonal CEA, although the importance of this observation is uncertain. Cytokeratin expression has also been described in EHE. 22.23

"Epithelioid haemangioendothelioma should be suspected in any lesion of pleural malignant mesothelioma, because the management and the prognosis of these two diseases are different"

The surgical treatment of EHE is complete excision or debulking of the tumour. Different adjuvant treatments have been proposed including interferon, interleukin 2, and retinoids. ${ }^{24}$ Regardless of the presence of the morphological features of EHE, most cases of pleural EHE behave much more aggressively than EHE arising from other sites. ${ }^{25}$

\section{ACKNOWLEDGEMENTS}

We wish to thank our senior electron microscopy technologist $S$ Ramjohn for his contribution.

\section{Authors' affiliations}

M Al-Shraim, D Ghazarian, Division of Pathology, Princess Margaret Hospital, Department of Laboratory Medicine and Pathobiology, University of Toronto, Toronto, Ontario, M5G2M9, Canada

B Mahboub, Division of Respirology, Department of Medicine, University of Toronto

P C Neligan, Division of Plastic Surgery, Department of Surgery, University of Toronto

D Chamberlain, Division of Pathology, Toronto General Hospital, Department of Laboratory Medicine and Pathobiology, University of Toronto

Correspondence to: Dr D Ghazarian, University Health Network, Princess Margaret Hospital, Suite 4-302, 610 University Avenue, Toronto, Ontario, M5G2M9, Canada; danny.ghazarian@uhn.on.ca

Accepted for publication 23 June 2004

\section{REFERENCES}

1 Weiss SF, Enzinger FM. Epithelioid hemangioendothelioma. A vascular tumor often mistaken for a carcinoma. Cancer 1982;50:970-81.

2 Verbeken E, Beyls J, Moerman P, et al. Lung metastasis of malignant epithelioid hemangioendothelioma mimicking a primary intravascular
Take home messages

- We report a case of primary pleural epithelioid haemangioendothelioma (EHE) with multiple metastases to the skin in a 51 year old man

- Pleural EHE can mimic malignant mesothelioma clinically, radiologically, and pathologically

- Immunohistochemical staining for vascular markers such as CD31, CD34, and factor VIII can help to distinguish EHE from mesothelioma

- Most pleural EHEs behave much more aggressively than EHE arising from other sites

- Some cases of EHE can be positive for monoclonal carcinoembryonic antigen, and this phenomenon requires further investigation

bronchioalveolar tumor. A histologic, ultrastructural, and immunohistochemical study. Cancer 1985:55:1741-6.

3 Yousem SA, Hochholzer L. Unusual thoracic manifestations of epithelioid hemangioendothelioma. Arch Pathol Lab Med 1987;1 11:459-63.

4 Quante M, Patel NK, Hill S, et al. Epithelioid hemangioendothelioma presenting in the skin: a clinicopathologic study of eight cases. Am J Dermatopathol 1998;20:541-6.

5 Churg A. Asbestos fibers and pleural plaques in a general autopsy population. Am J Pathol 1982;109:88-96.

6 Stephens M, Gibbs AR, Pooley FD, et al. Asbestos induced diffuse pleural fibrosis: pathology and mineralogy. Thorax 1987:42:583-8.

7 Wagner JC, Sloggs CA, Marchand P. Diffuse pleural mesotheliomas and asbestos exposure in a north western Cape province. $\mathrm{Br} J$ Ind Med 1960;17:260-71.

8 Attanoos RL, Suvarna SK, Rhead E, et al. Malignant vascular tumours of the pleura in "asbestos" workers and endothelial differentiation in malignant mesothelioma. Thorax 2000;55:860-3.

9 Crotty EJ, McAdams HP, Erasmus JJ, et al. Epithelioid hemangioendothelioma of the pleura: clinical and radiologic features. AJR Am J Roentgenol 2000; 175:1545-9.

10 Parums DV, Cordell JL, Micklem K, et al. JC 70: a new monoclonal antibody that detects endothelial associated antigen on routinely processed tissue sections. J Clin Pathol 1990;43:752-7.

11 Van de Rijn MRR. CD34. A review. Appl Immunohistochem 1994;2:71-80.

12 Guarda LA, Ordonez NG, Smith JL Jr, et al. Immunoperoxidase localization of factor VIII in angiosarcomas. Arch Pathol Lab Med 1982;106:515-16.

13 Just N, Tavernier JY, Salez F, et al. Multifocal epithelioid haemangioendothelioma: a difficult diagnosis. Rev Mal Respir 2003;20:761-5.

14 Vignon-Pennamen MD, Rybojad M, Verola O, et al. Epithelioid hemangioendothelioma: disease course in 3 cases. Ann Dermatol Venereol 1997; 124:165-6.

15 Wockel W, Haussinger K, Girgensohn S, et al. The morphology and clinical picture of epithelioid hemangioendothelioma of the lung. Dtsch Med Wochenschr 1991;116:1948-54.

16 Tomita M MY, Edagawa M, Maeda M, et al. Clinical and immunohistochemical study of eight cases with thymic carcinoma. BMC Surg 2002;23:2-3.

17 Ishida $\mathrm{H}$, Miwa $\mathrm{H}$, Tatsuta $\mathrm{M}$, et al. Ki-67 and CEA expression as prognostic markers in Dukes' C colorectal cancer. Cancer Lett 2004;207:109-15.

18 Golouh R, Vuzevski V, Bracko M, et al. Synovial sarcoma: a clinicopathological study of 36 cases. J Surg Oncol 1990;45:20-8.

19 Rosenberg AE, Brown GA, Bhan AK, et al. Chondroid chordoma-a variant of chordoma. A morphologic and immunohistochemical study. Am J Clin Pathol 1994;101:36-41.

20 Dalla Palma $\mathbf{P}$, Parenti $A$, Poletti $A$. CEA and $A B O(H)$ in upper urinary tract transitional tumors. Appl Pathol 1984;2:146-52.

21 Gray MH, Rosenberg AE, Dickersin GR, et al. Cytokeratin expression in epithelioid vascular neoplasms. Hum Pathol 1990;21:212-7.

22 Miettinen M, Fetsch JF. Distribution of keratins in normal endothelial cells and a spectrum of vascular tumors: implications in tumor diagnosis. Hum Pathol 2000;31:1062-7.

23 Billings SD, Folpe AL, Weiss SW. Epithelioid sarcoma-like hemangioendothelioma. Am J Surg Pathol 2003;27:48-57

24 Roudier-Pujol C, Enjolras O, Lacronique J, et al. Multifocal epithelioid hemangioendothelioma with partial remission after interferon alfa-2a treatment. Ann Dermatol Venereol 1994;121:898-904.

25 Zhang PJ, Livolsi VA, Brooks JJ. Malignant epithelioid vascular tumors of the pleura: report of a series and literature review. Hum Pathol 2000;31:29-34. 